\title{
Characterization of SuperCDMS 1-inch Ge Detectors
}

Z. Ahmed ${ }^{1}$, D.S. Akerib ${ }^{2}$, C.N. Bailey ${ }^{2}$, D. Balakishiyeva ${ }^{3}$, D.A. Bauer ${ }^{4}$, J. Beaty ${ }^{5}$, P.L. Brink ${ }^{6}$, R. Bunker ${ }^{7}$, B. Cabrera ${ }^{6}$, D.O. Caldwell ${ }^{7}$, K. Clark ${ }^{2}$, J. Cooley ${ }^{6}$, P. Cushman $^{5}$, F. DeJongh ${ }^{4}$, M.R. Dragowsky ${ }^{2}$, L. Duong ${ }^{5}$, E. Figueroa-Feliciano ${ }^{8}$, J. Filippini $^{9,1}$, M. Fritts ${ }^{5}$, S.R. Golwala ${ }^{1}$, D.R. Grant ${ }^{2}$, J. Hall ${ }^{4}$, R. Hennings-Yeomans ${ }^{2}$, S. Hertel ${ }^{8}$, D. Homgren ${ }^{4}$, L. Hsu ${ }^{4}$, M.E. Huber ${ }^{10}$, O. Kamaev ${ }^{5}$, M. Kiveni ${ }^{11}$, M. Kos ${ }^{11}$, S.W. Leman ${ }^{8}$, R. Mahapatra ${ }^{12}$, V. Mandic ${ }^{5}$, D. Moore ${ }^{1}$, K.A.

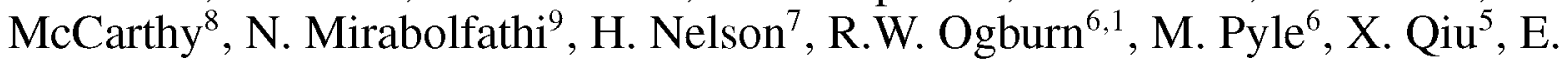
Ramberg $^{4}$, W. Rau ${ }^{13}$, A. Reisetter ${ }^{14,5}$, T. Saab ${ }^{3}$, B. Sadoulet ${ }^{15,9}$, J. Sander ${ }^{7}$, R.W. Schnee $^{11}$, D.N. Seitz ${ }^{9}$, B. Serfass ${ }^{9}$, K.M. Sundqvist ${ }^{9}$, A. Tomada ${ }^{6}$, G. Wang ${ }^{1}$, S. Yellin $^{6,7}$, J. Yoo ${ }^{4}$ and B.A. Young ${ }^{16}$

\footnotetext{
${ }^{1}$ Department of Physics, California Institute of Technology, Pasadena, CA 91125, USA

${ }^{2}$ Department of Physics, Case Western Reserve University, Cleveland, OH 44106, USA

${ }^{3}$ Department of Physics, University of Florida, Gainesville, FL 32611, USA

${ }^{4}$ Fermi National Accelerator Laboratory, Batavia, IL 60510, USA

${ }^{5}$ School of Physics \& Astronomy, University of Minnesota, Minneapolis, MN 55455, USA

${ }^{6}$ Department of Physics, Stanford University, Stanford, CA 94305, USA

${ }^{7}$ Department of Physics, University of California, Santa Barbara, CA 93106, USA

${ }^{8}$ Department of Physics, Massachusetts Institute of Technology, Cambridge, MA 02139, USA

${ }^{9}$ Department of Physics, University of California, Berkeley, CA 94720, USA

${ }^{10}$ Departments of Phys. \& Engr., University of Colorado Denver, Denver, CO 80217, USA

${ }^{11}$ Department of Physics, Syracuse University, Syracuse, NY 13244, USA

${ }^{12}$ Department of Physics, Texas A\&M University, College Station, TX 93106, USA

${ }^{13}$ Department of Physics, Queen's University, Kingston, ON, Canada, K7L $3 N 6$

${ }^{14}$ Department of Physics, Saint Olaf College, Northfield, MN 55057, USA

${ }^{15}$ Lawrence Berkeley National Laboratory, Berkeley, CA 94720, USA

${ }^{16}$ Department of Physics, Santa Clara University, Santa Clara, CA 95053, USA
}

\begin{abstract}
The newly commissioned SuperCDMS Soudan experiment aims to search for WIMP dark matter with a sensitivity to cross sections of $5 \times 10^{-45} \mathrm{~cm}^{2}$ and larger (90\% CL upper limit). This goal is facilitated by a new set of germanium detectors, 2.5 times more massive than the ones used in the CDMS-II experiment, and with a different athermal phonon sensor layout that eliminates radial degeneracy in position reconstruction of high radius events. We present characterization data on these detectors, as well as improved techniques for correcting position-dependent variations in pulse shape across the detector. These improvements provide surface-event discrimination sufficient for a reach of $5 \times 10^{-45} \mathrm{~cm}^{2}$.
\end{abstract}

Keywords: Dark Matter, Cryogenic Detectors, SuperCDMS

PACS: $95.35 .+\mathrm{d}, 14.80 . \mathrm{Ly}, 29.40 . \mathrm{Vj}, 73.61 . \mathrm{Jc}, 85.25 .-\mathrm{j}, 85.25 . \mathrm{Oj}$

\section{INTRODUCTION}

The Cryogenic Dark Matter Search Experiment (CDMSII) employs an array of germanium and silicon lowtemperature particle detectors to search for nuclear recoils from elastic scattering of Weakly Interacting Massive Particles (WIMPs). Particle interactions in these detectors yield ionization and athermal phonon signals, which we use to discriminate WIMP candidates from electromagnetic background. Past exposures of these detectors have yielded the world's leading limits on spin- independent interactions of WIMPs with nucleons over a large range of interesting WIMP masses $[1,2]$. The full exposure of CDMS-II is 2.5 times as large as that published to date, and is currently being analyzed.

The next phase of the experiment, SuperCDMS Soudan, will have a reach of $\sim 5 \times 10^{-45} \mathrm{~cm}^{2}$ in WIMPnucleon cross-section at $60 \mathrm{GeV} / \mathrm{c}^{2}$ of WIMP mass. An increase in per-detector mass by a factor of 2.5 combined with improved surface-event rejection will provide this reach by obtaining increased exposure with less than 1 event expected background [3]. 

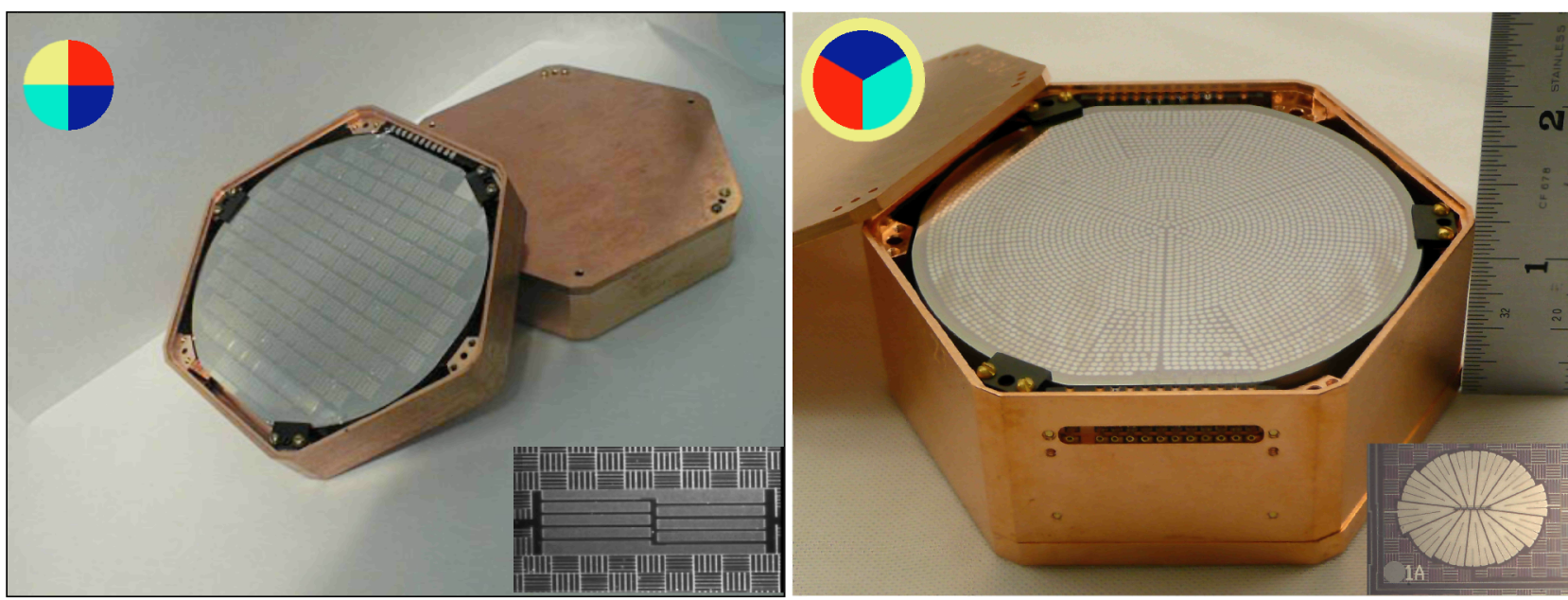

FIGURE 1. Left: A CDMS-II detector. Right: A SuperCDMS detector, 2.5 times more massive than the CDMS-II detectors, and with redesigned phonon sensors. Insets in both cases show phonon channel configuration and a zoomed-in view of a W TES and its Al phonon absorbers.

\section{DETECTOR DESIGN}

To obtain the necessary improvement in surface event rejection required for the larger SuperCDMS Soudan exposure, we undertook a redesign and systematically tested various detector design changes through several protoypes [4]. The final design consists of $2.5-\mathrm{cm}$ thick, 7.6$\mathrm{cm}$-diameter, 0.64-kg-mass germanium detectors that employ ionization and phonon sensors modified from the CDMS-II design, and are 2.5 times more massive than CDMS-II detectors. (See Fig. 1)

The CDMS-II detectors are $1-\mathrm{cm}$ thick germanium and silicon crystals with four quadrant-shaped phonon readout channels patterned on one face [5]. Each phonon channel contains tungsten Transition Edge Sensors (TES) connected to aluminum absorbers to sense athermal phonons from the crystal. An aluminum grid on the other face of the detector provides ionization readout and is divided into an outer guard ring and an inner grid to define a fiducial volume in the detector. The ionization yield, or the ratio of charge to phonon energy depositions, provides the primary discrimination between electron recoils and nuclear recoils. For events within $\sim 10 \mu \mathrm{m}$ of the surface of the detector, charge collection is suppressed and ionization yield is reduced. Additional discrimination against such events is obtained from the promptness of phonon pulses; surface events have faster pulses than bulk events.

The first step towards improving surface event discrimination is to increase the promptness of the phonon pulses for surface events. Thus, in the new design, we increased the surface-area coverage of the phonon-trapping fins for each TES from 19\% to 37\% [6]. This will allow more phonons to be collected prior to reflections off detector walls. The increase in coverage area was unaccompanied by an increase in the length that quasiparticles would have to traverse to find a TES, to prevent the phonon energy resolution from decreasing. Athermal phonon response is essentially independent of volume, so we do not expect a loss in energy resolution from the increased the detector thickness.

Next, we note observations from CDMS-II data that point to areas with potential for design changes leading to increased surface event discrimination. One of the effects of detector acoustics is phonon "foldback", the reflection of phonons off the cylindrical wall for highradius events in the detector. Consequently, the position for such events can be mis-reconstructed to low radius, if different parameters of the pulse shape are unable to break the degeneracy. CDMS-II data also indicate that there is a $4 \mu$ s gradient in timing information from the central to outer radius of the detector. This is comparable to timing difference between surface and bulk events. Thus, the foldback diminishes surface event discrimination in the outer-radial regions of the detector. For CDMS-II, these regions were excluded from WIMPsearch analysis.

For SuperCDMS, we attempt to mitigate the effect in the new design. The four phonon readout channels were rearranged from a quadrant configuration to a combination of an outer annulus and three inner triangular regions. This supplements the ionization guard ring, providing independent tagging of phonon foldback events and removing radial degeneracy of these events. Additionally, the coverage of the ionization guard ring was increased from $18 \%$ to $25 \%$ for a tighter fiducial volume cut. 


\section{DETECTOR TESTING AND CHARACTERIZATION}

We fabricated a prototype detector with these design modifications at the Stanford Nanofabrication Facility and performed a detailed characterization at the UC Berkeley Test Facility. We acquired calibration data using standard CDMS-II sources: ${ }^{133} \mathrm{Ba}$ and ${ }^{60} \mathrm{Co}$ for electron recoils, and ${ }^{252} \mathrm{Cf}$ for nuclear recoils. We observed that the phonon energy resolution of the prototype was similar to that of CDMS-II detectors, confirming that no loss mechanisms were introduced by modifying the phonon collection fins. Additionally, we saw that the increased detector thickness results in no degradation of ionization energy resolution, indicating that trapping of drifting charges remains negligible.

Next, we used the new information from the ratio of summed energy deposited in the inner sensors to that of the outer annular sensor to eliminate the radial degeneracy in reconstructing event positions (Fig. 2). We also noted that the prototype displays a higher correlation between depth information as deduced from pulse rise time and the manifold constructed from the radius variables in the plot. This indicates that event depth information in the prototype is less convolved with radial and angular position information compared to CDMS-II detectors.

Finally we tested the prototype's discrimination power between nuclear recoils and surface event background. To identify surface events, the test setup included $1-\mathrm{cm}$ ionization-only detectors above and below the prototype. Thus gamma calibration events with low yield in the prototype and coincident with an event in the top or bottom detectors would be tagged by the face of the prototype on which they occurred. Also, the ambient event rate in the absence of sources $(\sim 100 \mathrm{~Hz})$, was reduced by an order of magnitude by installing a $5-\mathrm{cm}$ thick lead shield around the ${ }^{3} \mathrm{He}-{ }^{4} \mathrm{He}$ dilution fridge. However, this led to a higher ambient neutron rate due to cosmogenic muons interacting in the lead shield. In the absence of a way to distinguish these neutrons from surface electron recoils in the nuclear recoil band, we were limited to evaluating rejection efficiency using a subset of surface electron recoils between the electron and nuclear recoil bands. Based upon previous data with CDMS-II detectors, these events should have phonon timing performance comparable to that of surface events at lower yields.

As shown in the left pane of Fig. 3, a CDMS-II style simple timing cut rejects a large fraction of the tagged surface events while maintaining good acceptance of nuclear recoils. To reject the remaining surface events, we invoke a new style of event discrimination developed during this analysis. The distribution and shape of phonon pulses can be used to create an $\mathrm{N}$-dimensional
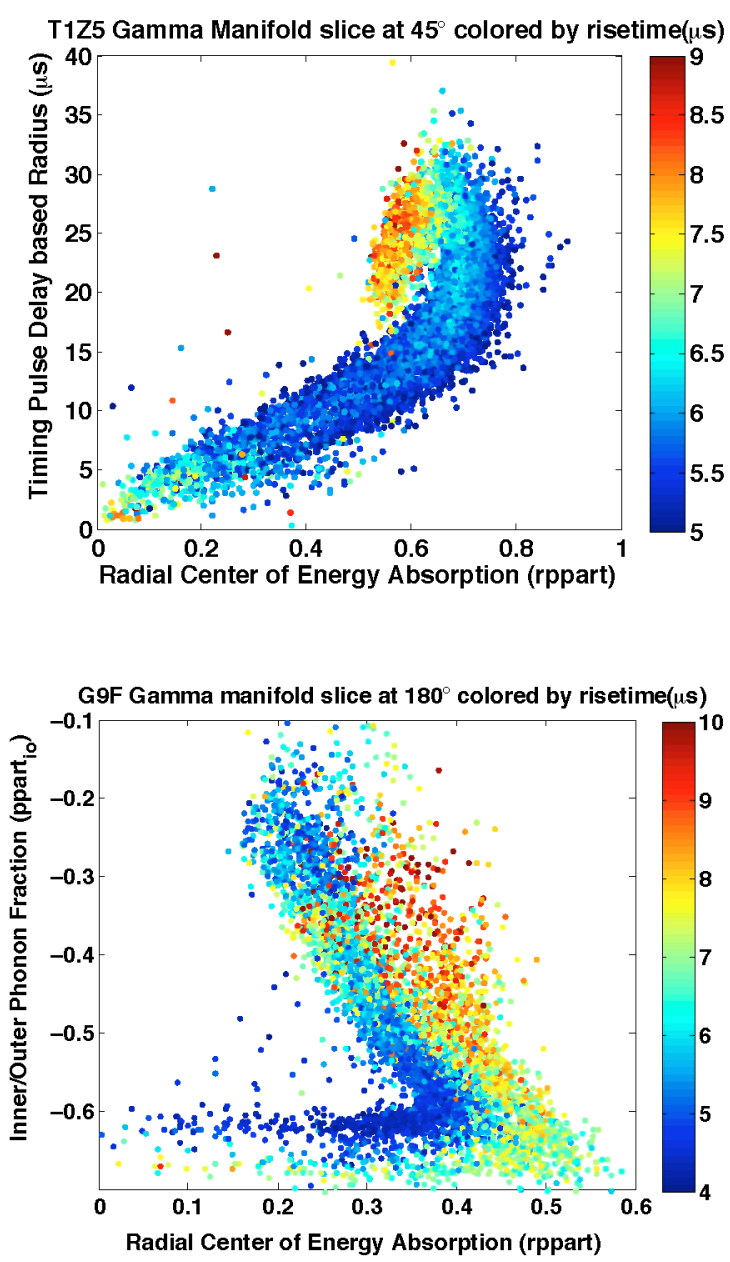

FIGURE 2. Top: Scatter plot of the phonon energy partition and phonon delay for CDMS-II detectors. The horizontal axis is the normalized radius measured using partition in energy between sensors. The vertical axis is the normalized radius from the relative delay between the phonon pulses compared to ionization. The scatter plot is colored by the rise time of the primary quadrant pulse for gamma calibration data in a very thin azimuthal slice of the detector. Bottom: Scatter plot of two phonon energy partition variables for the prototype detector. The horizontal axis gives the radius derived from the phonon partition among the three inner sensors and the vertical axis reports the energy partition between the summed inner phonon sensors and the outer annular phonon sensor. The scatter plot is colored by the rise time of the phonon pulses from the primary phonon sensor.

manifold from $\mathrm{N}$ measured quantities. These $\mathrm{N}$ observables are not only functions of the energy and its center of deposition, but also of the type of recoil; the characteristics of this manifold are different for electron recoils and nuclear recoils. So, for each event we construct two $\chi^{2}$ parameters measuring the compatibility with a nuclear recoil hypothesis and with an electron recoil hypothesis. 

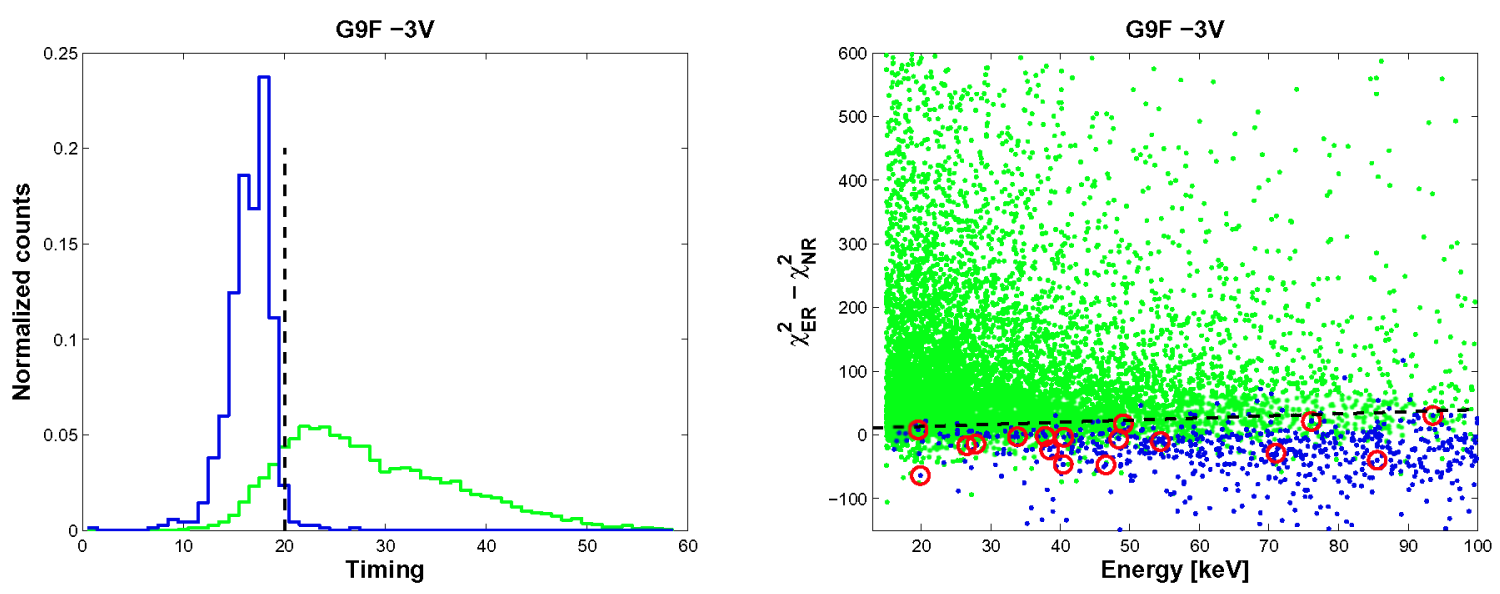

FIGURE 3. Left: Histogram of timing parameter distribution for nuclear recoil events (green) and tagged surface electron recoil events (blue). The suggested cut is indicated by a dashed line. A simple timing parameter cut will remove almost all surface events. Right: Distribution of the surface events (blue) and nuclear recoil events (green) in the $\chi_{E R}^{2} \chi_{N R}^{2}$ and energy parameter space. The events which pass the timing cut are circled in red and rejected by the cut below the dashed line.

The difference of the two $\chi^{2}$ gives a new discriminator using which we can reject the remaining surface events as shown in the right pane of Fig. 3. We can put a $90 \%$ confidence upper limit on the combined timing and $\chi^{2}$ rejection of $2.3 / 733(\sim 0.3 \%)$ at a passage fraction of $77.5 \%$. This surpasses $0.25 \%$ rejection at $60 \%$ passage fraction, the goal for the SuperCDMS detectors.

These results sufficed in designating the design for this prototype as the baseline design for SuperCDMS Soudan detectors. Production of the first "SuperTower" (ST-1), a stack consisting of five 1-inch thick Ge detectors and two endcap ionization-only veto detectors hence proceeded. ST-1 was subsequently installed in the Soudan Underground Laboratory, replacing two CDMS-II towers, and base temperature was reached on June 4, 2009. ST-1 is now operational, and is being tuned as of this writing. Calibration with radioactive sources and WIMP-search data acquisition will commence shortly.

\section{CONCLUSION}

A redesign of CDMS-II detectors to meet the objectives of SuperCDMS Soudan was successfully completed, resulting in a new baseline design for 1-inch thick germanium detectors. We tested a prototype at the UC Berkeley Test Facility and have shown energy resolution comparable to CDMS-II detectors. Also, we have demonstrated enhanced surface event background discrimination resulting from better reconstruction of event position and interaction type using phonon pulse shape characteristics. This successful design has been used to produce the first set of science detectors for SuperCDMS Soudan which will start taking WIMP-search data in the next few weeks.

\section{ACKNOWLEDGMENTS}

This work is supported in part by the National Science Foundation (Grant Nos. AST-9978911, PHY- 0542066, PHY-0503729, PHY-0503629, PHY-0503641, PHY0504224, PHY-0705052, PHY-0801536, PHY-0801708, PHY-0801712 and PHY-0802575), by the Department of Energy (Contracts DE-AC03-76SF00098, DE-FG02-91ER40688, DE-FG02-92ER40701, DEFG03-90ER40569, and DE-FG03-91ER40618), and by NSERC Canada (Grant SAPIN 341314-07).

\section{REFERENCES}

1. D.S. Akerib, et al., Phys. Rev. Lett. 93, 211301 (2004).

2. Z. Ahmed, et al., Phys. Rev. Lett. 102, 011301 (2009).

3. D.S. Akerib, et al., Nucl. Instr. Methods A 559 (2006).

4. C.N. Bailey, et al., these proceedings, 2009.

5. D.S. Akerib et al., Phys. Rev. D 72, 052009 (2005)

6. C.N. Bailey, et al., J. Low Temp. Phys. 151 (2008). 